\title{
Optical thin-film materials with low refractive index for broadband elimination of Fresnel reflection
}

\author{
J.-Q. XI ${ }^{1,2}$, MARTIN F. SCHUBERT 1,3 , JONG KYU KIM ${ }^{1,3}$, E. FRED SCHUBERT ${ }^{1,2,3 *}$, MINFENG CHEN ${ }^{1,4}$, \\ SHAWN-YU LIN ${ }^{1,2}$, W. LIU ${ }^{5}$ AND J. A. SMART ${ }^{5}$
}

${ }^{1}$ Future Chips Constellation, Rensselaer Polytechnic Institute, 110 8th Street, Troy, New York 12180, USA

2Department of Physics, Applied Physics, and Astronomy, Rensselaer Polytechnic Institute, 110 8th Street, Troy, New York 12180, USA

${ }^{3}$ Department of Electrical, Computer, and Systems Engineering, Rensselaer Polytechnic Institute, 110 8th Street, Troy, New York 12180, USA

${ }^{4}$ Graduate Institute of Electro-Optical Engineering, National Taiwan University, Taipei, Taiwan, R.O.C.

${ }^{5}$ Crystal IS, Inc., Green Island, New York 12183, USA

*e-mail: EFSchubert@rpi.edu

In 1880, by studying light passing through Earth's atmosphere, Lord Rayleigh mathematically demonstrated that graded-refractiveindex layers have broadband antireflection characteristics ${ }^{1}$. Graded-index coatings with different index profiles have been investigated for broadband antireflection properties, particularly with air as the ambient medium ${ }^{2-4}$. However, because of the unavailability of optical materials with very low refractive indices that closely match the refractive index of air, such broadband antireflection coatings have not been realizable. Here we report the fabrication of $\mathrm{TiO}_{2}$ and $\mathrm{SiO}_{2}$ graded-index films deposited by oblique-angle deposition, and, for the first time, we demonstrate their potential for antireflection coatings by virtually eliminating Fresnel reflection from an AlN-air interface over a broad range of wavelengths. This is achieved by controlling the refractive index of the $\mathrm{TiO}_{2}$ and $\mathrm{SiO}_{2}$ nanorod layers, down to a minimum value of $n$ $=1.05$ in the case of the latter, the lowest value so far reported.

In optical sciences, the refractive index of a medium, first considered by Isaac Newton, is the most fundamental quantity. The refractive index of a medium determines not only the phase velocity of light, but also refraction, reflection and diffraction occurring at the boundary of the medium. In 1621, Willebrord Snell discovered the sinusoidal relationship between the angle of incidence and the angle of refraction when a light ray passes from one optical medium to another ${ }^{5}$. In 1821, Augustin Jean Fresnel presented the laws that allow us to calculate the intensity of polarized light reflected and refracted at the boundary between two media with different refractive indices ${ }^{6}$.

Unfortunately, the availability of materials with suitable refractive indices is very limited, particularly for optical films with refractive indices less than 1.4. Silica $\left(\mathrm{SiO}_{2}\right)$ and magnesium fluoride $\left(\mathrm{MgF}_{2}\right)$ have refractive indices of 1.46 and 1.39 , respectively. Dense materials with a refractive index of, say, 1.10 or 1.20 , do not exist. However, such materials would be highly desirable for many applications, for example, broadband antireflection coatings with air-ambient ${ }^{2-4}$, omnidirectional reflectors ${ }^{7,8}$, distributed Bragg reflectors ${ }^{9,10}$, optical microresonators ${ }^{11}$, light-emitting diodes ${ }^{12}$ and optical interconnects ${ }^{13}$.
Recently, we reported the fabrication of nanostructured porous $\mathrm{SiO}_{2}$ with a low refractive index of 1.08 (ref. 14). It was shown that oblique-angle deposition is a viable technique for the deposition of high-quality, low-refractive-index (low- $n)$ materials ${ }^{15}$. Here, an even lower value of $n=1.05$ is reported for $\mathrm{SiO}_{2}$ grown by oblique-angle deposition, with a vapour incident angle of $\theta_{\mathrm{v}}=87^{\circ}$. Furthermore, we demonstrate the feasibility of a wellcontrolled graded-index profile using low- $n \mathrm{TiO}_{2}$ and $\mathrm{SiO}_{2}$ materials. Figure la shows a cross-sectional scanning electron micrograph (SEM) of the low- $n$ material. It consists of an array of $\mathrm{SiO}_{2}$ nanorods with a tilt angle of $\theta_{\mathrm{t}}=45^{\circ}$. The feature size of the low- $n$ material is much smaller than the wavelength of visible light, so Mie and Rayleigh scattering can be neglected. Figure 1b shows the dispersion curve of the $\mathrm{SiO}_{2}$ low- $n$ material measured by ellipsometry. The thin-film thickness measured by ellipsometry was confirmed by SEM. Oblique-angle deposited films have been reported to be anisotropic ${ }^{16}$. We do find some optical anisotropy for nanorod layers with refractive indices of $n \gg 1.0$. However, no significant anisotropy is found in our nanorod films, which have a refractive index of $n \approx 1.0$. Next, we will show that the extremely low refractive index allows us to virtually eliminate Fresnel reflection over a broad wavelength and angleof-incidence range.

Conventional single-layer antireflection coatings, although widely used, work only at a single wavelength and at normal incidence. However, graded-index coatings yield omnidirectional, broadband antireflection characteristics. Figure $2 \mathrm{a}$ shows three different graded-index profiles with linear, cubic and quintic profiles for a substrate with $n_{\mathrm{s}}=2.05$ (ref. 2). The calculated spectral and angular dependences of the reflectivity for these three index profiles are shown in Fig. $2 b$ and c, respectively. Each graded-index profile exhibits low reflectivity for both transverse electric (TE) and transverse magnetic (TM) polarizations over a broad spectral range, with the quintic-index profile having the best performance, with $R<0.1 \%$ for the entire visible spectrum. It has been reported that the quintic-index profile is near the optimum profile for a graded-index antireflection coating ${ }^{2,17}$. 

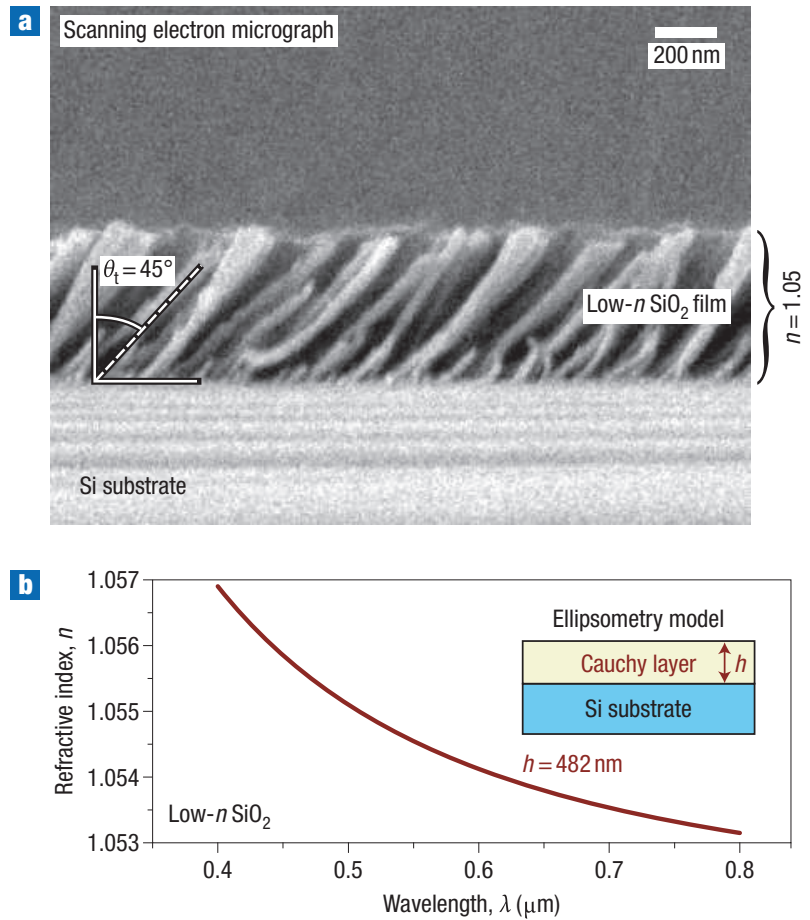

Figure 1 Low- $\boldsymbol{n}$ material. a, SEM image of a low- $n \mathrm{SiO}_{2}$ nanorod thin film with $\theta_{\mathrm{t}}=45^{\circ}$. b. Cauchy-type dispersion curve of low- $n \mathrm{SiO}_{2}$ nanorod thin film measured by ellipsometry.

Fresnel reflection can be eliminated by the modified-quintic-index profile for omnidirectional incidence over a broad wavelength range $^{3,4}$. Importantly, all of these profiles require optical materials with refractive indices that span the range from air to substrate index. Note that the design principles presented here can be applied to any substrate material.

Different techniques have been reported for producing graded-index coatings with air ambient, including a chemical etch-leach process on a glass surface ${ }^{18-20}$, the replication of a rough surface ${ }^{21}$, interference-patterning by two coherent light beams ${ }^{22}$, and a recently reported sol-gel process ${ }^{23}$. However, none of these techniques has good control over the refractiveindex profile. Here, we report a graded-index broadband, omnidirectional antireflection coating using physical vapour deposition. This technique yields excellent control over the refractive-index profile.

Oblique-angle deposition is a vapour-deposition technique widely used to grow nanostructured thin-film materials with controllable porosity ${ }^{24-26}$, which is achieved by a self-shadowing effect and surface diffusion ${ }^{27,28}$. Optical thin films consisting of nanorods grown by oblique-angle deposition using electron-beam evaporation have a lower refractive index than dense materials due to the nano-porous nature of the material. Our experiments show that the refractive index of a $\mathrm{TiO}_{2}$ nanorod layer can be controllably varied from 2.7 to 1.3 by changing the vapour incident angle. The refractive index of a $\mathrm{SiO}_{2}$ nanorod layer can be varied from 1.46 to 1.05 . Therefore, the combination of $\mathrm{TiO}_{2}$ and $\mathrm{SiO}_{2}$ nanorod layers can be used to achieve any refractiveindex value between 2.7 and 1.05. A graded-index coating consisting of $\mathrm{TiO}_{2}$ and $\mathrm{SiO}_{2}$ nanorod layers grown by obliqueangle deposition using electron-beam evaporation on a oneside-polished AlN substrate $\left(n_{\mathrm{s}}=2.05\right)$ is demonstrated to have a

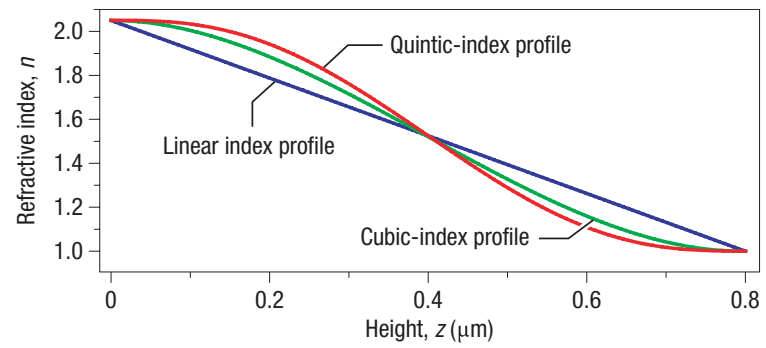

b

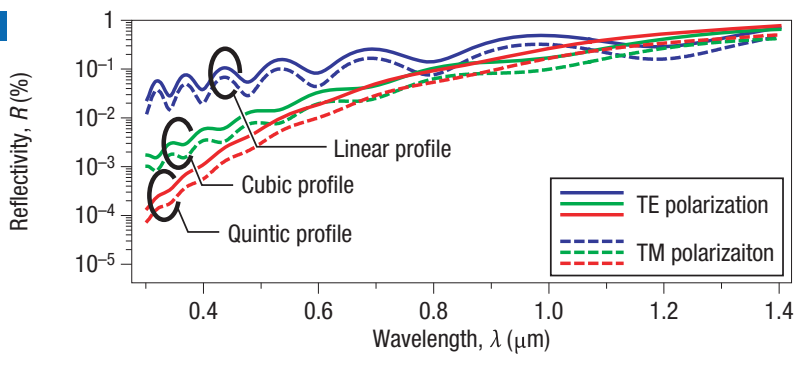

0

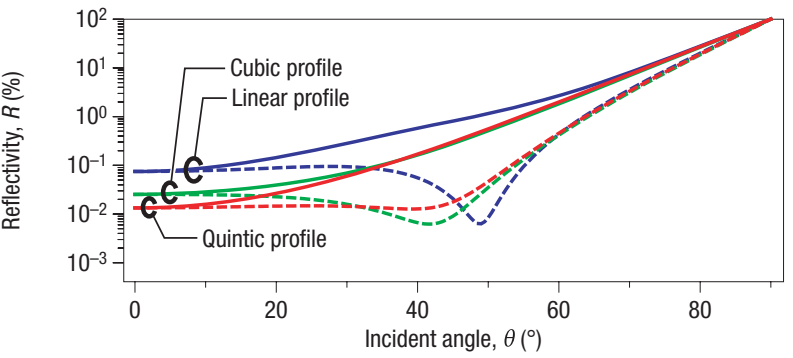

Figure 2 Different index profiles for graded-index coating. a, Linear-, cubicand quintic-index profiles that have index matching with air and a substrate with refractive index of $n_{\mathrm{s}}=2.05$. $\mathbf{b}$, Calculated wavelength-dependent reflectivities for linear-, cubic- and quintic-index profiles at normal incidence. c, Calculated angular-dependent reflectivities for linear-, cubic- and quintic-index profiles at a wavelength $\lambda=632.8 \mathrm{~nm}$. The calculation is approximated by using 1,000 sublayers of equal thickness.

near-perfect antireflection characteristics. The coating consists of five layers with refractive indices following the modified-quinticindex profile. Figure $3 \mathrm{a}$ is a cross-sectional SEM of the coating, clearly showing the stack of $\mathrm{TiO}_{2}$ and $\mathrm{SiO}_{2}$ nanorod layers. The lower three layers are made of $\mathrm{TiO}_{2}$ nanorods, and the upper two layers are made of $\mathrm{SiO}_{2}$ nanorods. All layers have well-defined interfaces, and the feature size of individual nanorods is smaller than $50 \mathrm{~nm}$, that is, much smaller than the wavelength of visible light. The low- $n$ films are found to be stable, with no evidence of degradation, for example, due to the adsorption of moisture. This finding is consistent with the literature, which reports oblique-angle deposited films to be robust to micromachining ${ }^{29}$.

The thickness and the refractive index of each layer, measured by ellipsometry, are listed, along with the vapour incident angle, in Table 1. The thicknesses are confirmed by SEM as shown in Fig. 3a. $\mathrm{TiO}_{2}$ is deposited by reactive electron-beam evaporation with an $\mathrm{O}_{2}$ partial pressure of $2 \times 10^{-4}$ torr (ref. 30). The increasing vapour incident angle for successive layers prevents the filling-in of space between nanorods of the previous deposition. For the same reason, the first $\mathrm{SiO}_{2}$ nanorod layer is deposited on the $\mathrm{TiO}_{2}$ nanorod layer with a negative vapour incident angle. The SEM micrograph of Fig. 3a shows no fill-in effect at the interfaces. The surface of a single low- $n$ layer is optically specular. 


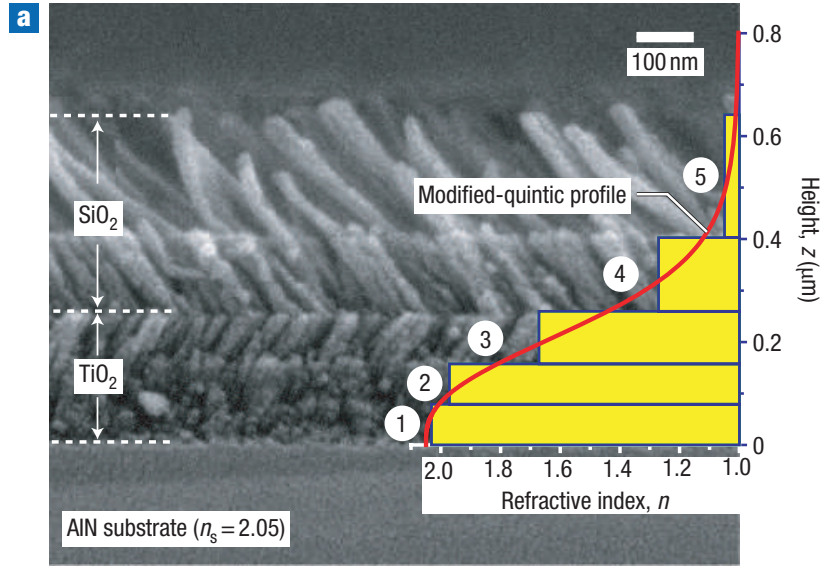

b

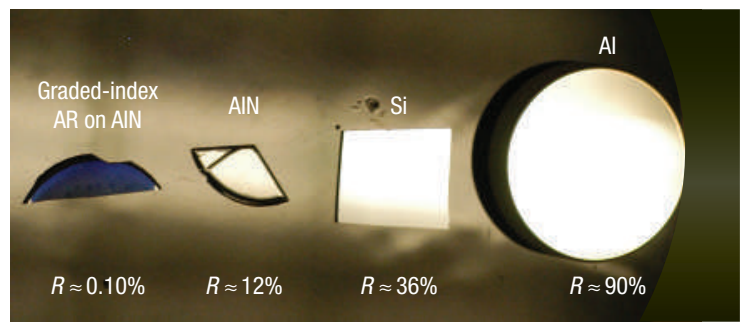

Figure $3 \mathrm{TiO}_{2}-\mathrm{SiO}_{2}$ graded-index coating. a, Cross-sectional SEM image of graded-index coating with a modified-quintic-index profile. The graded-index coating consists of three $\mathrm{TiO}_{2}$ nanorod layers and two $\mathrm{SiO}_{2}$ nanorod layers.

b. Photograph of a graded-index antireflection coating on AIN and the specular surfaces of AIN, Si and Al.

The bottom layer of the thin-film structure has a refractive index of 2.03, which closely matches the index of AlN. The top layer has a refractive index of 1.05 , very close to the index of air. Therefore, the thin-film structure matches the refractive indices of air and the substrate and has excellent antireflection characteristics. The photograph in Fig. 3b shows the graded-index coating on AlN in comparison with the surfaces of other materials. The graded-index coating exhibits very low reflectivity, with values as small as $R=0.1 \%$. The $\mathrm{Al}$-air, $\mathrm{Si}-$ air and AlN-air interfaces, on the other hand, have reflectivities of $R \approx 90 \%, R \approx 36 \%$ and $R \approx 12 \%$, respectively. In the photograph, the graded-index coating on the AlN substrate exhibits a very dark surface, confirming the absence of Fresnel reflection over a broad spectral width.

The angular-dependent reflectivity of the graded-index coating on the AlN substrate is measured at $\lambda=632.8 \mathrm{~nm}$ using a $\mathrm{He}-\mathrm{Ne}$

Table 1 Vapour source material, vapour incident angle in oblique-angle deposition, measured refractive index and thickness of layers forming the graded-index coating.

\begin{tabular}{llllll}
\hline \multicolumn{7}{c}{ Graded-index layer number } \\
\cline { 2 - 6 } & 1 & 2 & 3 & 4 & 5 \\
\hline Vapour source & $\mathrm{TiO}_{2}$ & $\mathrm{TiO}_{2}$ & $\mathrm{TiO}_{2}$ & $\mathrm{SiO}_{2}$ & $\mathrm{SiO}_{2}$ \\
Vapour incident angle (deg) & 25 & 40 & 65 & -68 & -87 \\
Measured thickness (nm) & 77.4 & 80.2 & 99.3 & 145.0 & 223.0 \\
Measured refractive index & 2.03 & 1.97 & 1.67 & 1.27 & 1.05 \\
\hline $\begin{array}{l}\text { The thickness and the refractive index for each layer are measured by ellipsometry. The refractive indices are the } \\
\text { ones at a wavelength of } \lambda=632.8 \mathrm{~nm} .\end{array}$
\end{tabular}
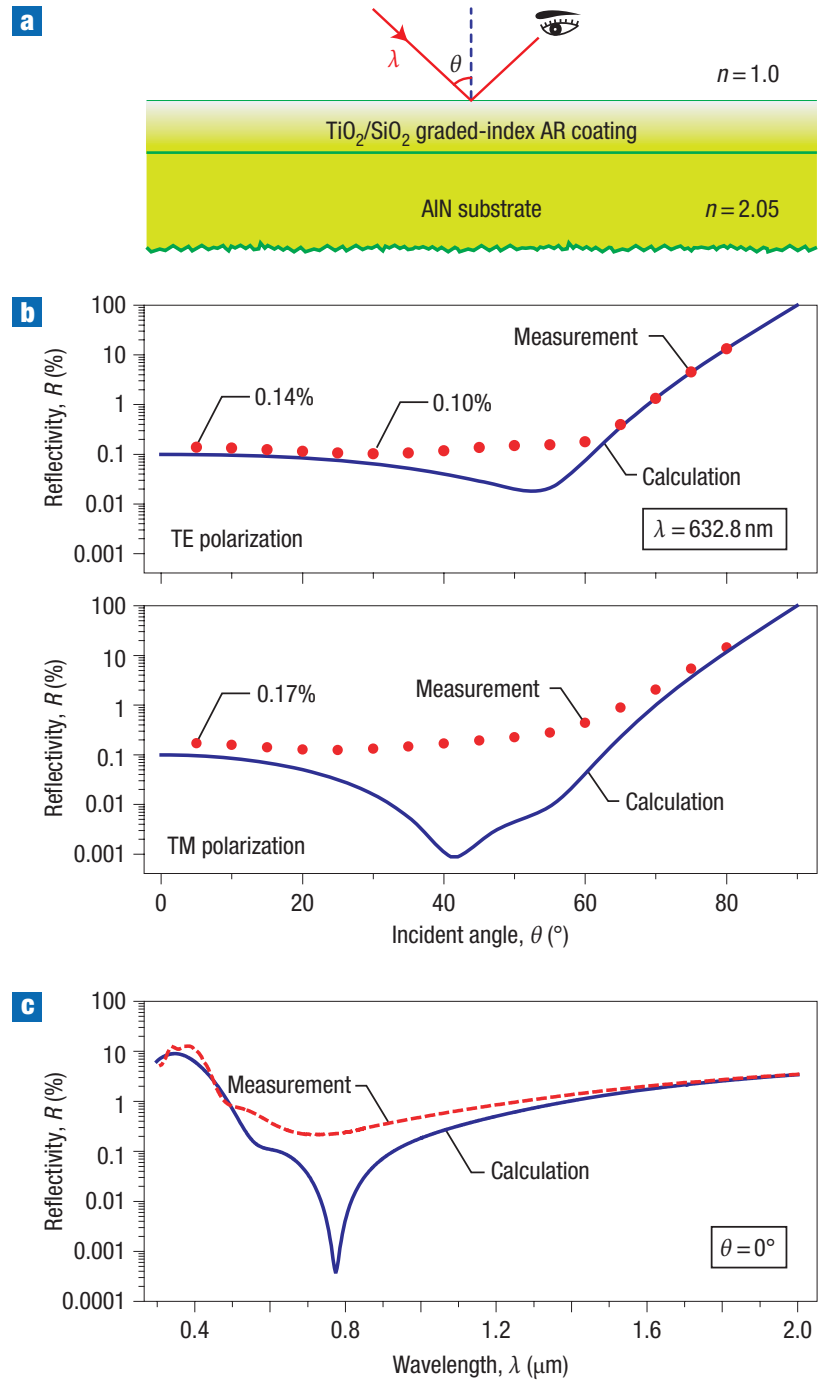

Figure 4 Reflectivity of graded-index coating. a, Schematic of reflectivity measurement. b, Theoretical (solid line) and measured (dotted line) reflectivity versus incident angle of graded-index coating at wavelength $\lambda=632.8 \mathrm{~nm}$. c, Wavelength dependence of theoretical (solid line) and measured (dashed line) reflectivity of graded-index coating at normal incidence.

laser as the light source. A schematic of the setup is shown in Fig. 4a. Figure $4 \mathrm{~b}$ shows the experimental measurement and theoretical results for TE and TM polarizations. The measurements and the calculations are in very good agreement. The graded-index coating has very low reflectivity for all incident angles except for angles close to $90^{\circ}$. The lowest reflectivity from this measurement is $R=0.1 \%$ at an incident angle of $30^{\circ}$ for $\mathrm{TE}$ polarization. At an incident angle of $\theta=5^{\circ}$, the measured reflectivity is $0.14 \%$ for $\mathrm{TE}$ polarization and $0.17 \%$ for $\mathrm{TM}$ polarization. For angles ranging from $0^{\circ}$ to $55^{\circ}$, the reflectivity is measured to be $<0.3 \%$ for both polarizations. The wavelengthdependent reflectivity is measured at normal incidence using a spectrophotometer over a wavelength range from $0.3 \mu \mathrm{m}$ to 2 $\mu \mathrm{m}$. Theoretical calculations and measurements agree, as shown in Fig. 4c. The results demonstrate that the graded-index coating has low reflectivity over the entire visible and near-infrared spectrum. The reflectivity is measured to be $<0.5 \%$ at wavelengths ranging from $574 \mathrm{~nm}$ to $1,010 \mathrm{~nm}$. At a wavelength 
around $400 \mathrm{~nm}$, the reflectivity of the graded-index coating rises, which explains the dark-bluish colour of the coating seen in Fig. 3b. The angular dependence and spectral dependence of the reflectivity confirm the broadband, omnidirectional nature of the graded-index coating. Note that the reflection from the roughened back side of the AlN substrate cannot be eliminated in the reflectivity measurement, and is probably the reason for the residual reflectivity.

In conclusion, a low-refractive-index optical thin film with the unprecedented refractive index of $n=1.05$ is demonstrated. A graded-index coating consisting of $\mathrm{TiO}_{2}$ and $\mathrm{SiO}_{2}$ nanorod layers is successfully demonstrated to virtually eliminate Fresnel reflection over a broad range of wavelengths. The lowest reflectivity measured is $R=0.10 \%$. The reflectivity remains below $0.3 \%$ for incident angles between $0^{\circ}$ and $55^{\circ}$ at $\lambda=632.8 \mathrm{~nm}$. For normal incidence, the reflectivity is found to be less than $0.5 \%$ at wavelengths from $574 \mathrm{~nm}$ to $1,010 \mathrm{~nm}$.

\section{METHODS}

Our nanorod thin films are grown by oblique-angle deposition using electronbeam evaporation. The vapour-source materials are $\mathrm{SiO}_{2}$ for $\mathrm{SiO}_{2}$ nanorod layers and $\mathrm{Ti}_{3} \mathrm{O}_{5}$ for $\mathrm{TiO}_{2}$ nanorod layers. The apparatus used in our oblique-angle deposition has a sample stage, on which the substrate is loaded, with controllable polar-angle rotation. The distance between substrate and the source material is $28 \mathrm{~cm}$. During the deposition, $\mathrm{O}_{2}$ is supplied at a partial pressure of $2 \times 10^{-4}$ torr, and there is no movement of the substrate. For each layer, the sample stage is at a fixed polar angle so that the substrate has a certain tilt angle with respect to the vapour-flux direction as listed in Table 1 . The tilt angle of the substrate is only changed between deposition runs of different layers. Before the $\mathrm{SiO}_{2}$ layer is deposited on the $\mathrm{TiO}_{2}$ layer, the substrate is rotated to the opposite direction so that the $\mathrm{SiO}_{2}$ nanorods and $\mathrm{TiO}_{2}$ nanorods are approximately orthogonal to each other, as illustrated in Fig. 3a.

Received 23 November 2006; accepted 24 January 2007; published 1 March 2007.

\section{References}

1. Rayleigh, J. S. On reflection of vibrations at the confines of two media between which the transition is gradual. Proc. London Math. Soc. 11, 51-56 (1880).

2. Southwell, W. H. Gradient-index antireflection coatings. Opt. Lett. 8, 584-586 (1983)

3. Dobrowolski, J. A., Poitras, D., Ma, P., Vakil, H. \& Acree, M. Toward perfect antireflection coatings: numerical investigation. Appl. Opt. 41, 3075-3083 (2002).

4. Poitras, D. \& Dobrowolski, J. A. Toward perfect antireflection coatings. 2. Theory. Appl. Opt. 43, 1286-1295 (2004).

5. Vollgraff, J. A. Snellius' notes on the reflection and refraction of rays. Osiris 1, 718-725 (1936).

6. Boutry, G. A. Augustin Fresnel: His time, life and work 1788-1827. Science Progress 36, 587-604 (1948).
7. Xi, J.-Q. et al. Internal high reflectivity omni-directional reflectors. Appl. Phys. Lett. 87, 031111 (2005).

8. Xi, J.-Q. et al. Omnidirectional reflector using nanoporous $\mathrm{SiO}_{2}$ as a low-refractive-index material. Opt. Lett. 30, 1518-1520 (2005).

9. Sharma, R., Haberer, E. D., Meier, C., Hu, E. L. \& Nakamura, S. Vertically oriented GaN-based air-gap distributed Bragg reflector structure fabricated using band-gap-selective photoelectrochemical etching. Appl. Phys. Lett. 87, 051107 (2005).

10. Ho, S.-T. et al. High index contrast mirrors for optical microcavities. Appl. Phys. Lett. 57, 1387-1389 (1990).

11. Xu, Q., Almeida, V. R., Panepucci, R. R. \& Lipson, M. Experimental demonstration of guiding and confining light in nanometer-size low-refractive-index material. Opt. Lett. 29, 1626-1628 (2004).

12. Kim, J. K. et al. GaInN light-emitting diode with conductive omnidirectional reflector having a low-refractive-index indium-tin oxide layer. Appl. Phys. Lett. 88, 013501 (2006).

13. Jain, A. et al. Porous silica materials as low- $k$ dielectrics for electronic and optical interconnects. Solid Thin Films 398-399, 513-522 (2001).

14. Xi, J.-Q., Kim, J. K. \& Schubert, E. F., Silica nanorod-array films with very low refractive indices. Nano Lett. 5, 1385-1387 (2005).

15. Xi, J.-Q. et al. Very low-refractive-index optical thin films consisting of an array of $\mathrm{SiO}_{2}$ nanorods. Opt. Lett. 31, 601-603 (2006)

16. Hodgkinson, I. J., Horowitz, F., Macleod, H. A., Sikkens, M. \& Wharton, J. J. Measurement of the principal refractive indices of thin films deposited at oblique incidence. J. Opt. Soc. Am. A 2, 1693-1697 (1985)

17. Southwell, W. H. Pyramid-array surface-relief structures producing antireflection index matching on optical surfaces. J. Opt. Soc. Am. A 8, 549-553 (1991).

18. Minot, M. J. Single-layer, gradient refractive index antireflection films effective from $0.35 \mu \mathrm{m}$ to $2.5 \mu \mathrm{m}$. J. Opt. Soc. Am. 66, 515-519 (1976).

19. Asahara, Y. \& Izumitani, T. The properties of gradient index antireflection layer on the phase separable glass. J. Non-Crys. Solids 42, 269-279 (1980).

20. Yoldas, B. E. \& Partlow, D. P. Wide spectrum antireflective coating for fused silica and other glasses. Appl. Opt. 23, 1418-1424 (1984)

21. Maffitt, K. N., Brueckner, H. U. \& Lowrey, D. R. Polymeric optical element having antireflecting surface. US Patent 4,153,654, 8 May 1979.

22. Wilson, S. J. \& Hutley, M. C. The optical properties of "moth eye" antireflection surfaces. Opt. Acta 7, 993-1009 (1982).

23. Wu, G. et al. Preparation and properties of scratch-resistant nano porous broadband AR silica films derived by a two-step catalytic sol-gel process. Proc. SPIE 4086, 807-810 (2000).

24. Robbie, K. \& Brett, M. J. Sculptured thin films and glancing angle deposition: Growth mechanics and applications. J. Vac. Sci. Technol. A 15, 1460-1465 (1997).

25. Robbie, K. et al. Ultrahigh vacuum glancing angle deposition system for thin films with controlled three-dimensional nanoscale structure. Rev. Sci. Instrum. 75, 1089-1097 (2004).

26. Kennedy, S. R. \& Brett, M. J. Porous broadband antireflection coating by glancing angle deposition. Appl. Opt. 42, 4573-4579 (2003)

27. Robbie, K., Sit, J. C. \& Brett, M. J. Advanced techniques for glancing angle deposition. J. Vac. Sci. Technol. B 16, 1115-1122 (1998).

28. Abelmann, L. \& Lodder, C. Oblique evaporation and surface diffusion. Thin Solid Films 305, $1-21$ (1997).

29. Harris, K. D., Westra, K. L. \& Brett, M. J. Fabrication of perforated thin films with helical and chevron pore shapes. Electrochem. Solid-State Lett. 4, C39-C42 (2001).

30. Pulker, H. K., Paesold, G. \& Ritter, E. Refractive indices of $\mathrm{TiO}_{2}$ films produced by reactive evaporation of various titanium-oxygen phases. Appl. Opt. 15, 2986-2991 (1976).

\section{Acknowledgements}

The authors gratefully acknowledge support from Sandia National Laboratories (USA), Crystal IS Corporation (USA), Samsung Advanced Institute of Technology (Korea), the Army Research Office (USA), the New York State Office of Science, Technology and Academic Research (USA), the National Science Foundation (USA) and the Department of Energy (USA).

Correspondence and requests for materials should be addressed to E.F.S.

Competing financial interests

The authors declare that they have no competing financial interests.

Reprints and permission information is available online at http://npg.nature.com/reprintsandpermissions/ 
Reproduced with permission of the copyright owner. Further reproduction prohibited without permission. 